\title{
Féeries
}

Études sur le conte merveilleuX, XVII -XIXe siècle

Contes et morale(s)

\section{Les anges déchus qui font rire}

Fallen Angels that Make You Laugh

\section{Aboubakr Chraïbi}

\section{OpenEdition}

\section{Journals}

Édition électronique

URL : http://journals.openedition.org/feeries/1008

ISSN : 1957-7753

Éditeur

UGA Éditions/Université Grenoble Alpes

Édition imprimée

Date de publication : 15 octobre 2016

Pagination : 165-182

ISBN : 978-2-8310-335-3

ISSN : 1766-2842

\section{Référence électronique}

Aboubakr Chraïbi, « Les anges déchus qui font rire », Féeries [En ligne], 13 | 2016, mis en ligne le 01 janvier 2017, consulté le 08 septembre 2020. URL : http://journals.openedition.org/feeries/1008

(C) Féeries 


\section{LES ANGES DÉCHUS QUI FONT RIRE}

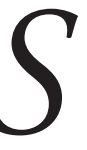

I LES ORIGINES MULTIPLES MAIS GLOBALEMENT ORIENTALES des Mille et Un Jours de Pétis de La Croix ne font pas de doute, il reste difficile d'en fournir tout le détail. De ce point de vue, Paul Sebag a réalisé un travail remarquable, actualisé de manière non moins remarquable par Raymonde Robert ${ }^{\mathrm{I}}$. Paul Sebag a notamment permis de faire la part des différentes sources turques utilisées par l'orientaliste français, entre le Faraj ba'd al-shidda (La délivrance après la difficulté), comme source principale, et les Quarante vizirs, comme ouvrage d'appoint ${ }^{2}$. Les Mille et Un Jours comportent aussi une part d'invention, qui peut s'exprimer à travers des mélanges inédits, l'éclatement de la première histoire pour en faire un récit-cadre, l'invention de l'intitulé du livre, et, peu nombreuses il est vrai, quelques zones d'ombre, quelques contes dont on ne sait s'ils ont été entièrement créés par l'orientaliste, sous couvert de traduction, ou s'ils sont l'adaptation de quelque tradition narrative arabe, turque, persane ou indienne.

L'un de ces récits aux origines indéterminées, l'Histoire des deux frères génies Adis et Dahy, semble à la fois s'ancrer dans un drame mythologique archaïque et prétendre à la légèreté d'une comédie mettant en scène de riches vieillards tentant sans succès de séduire de jolies orphelines ${ }^{3}$. Comme Christelle Bahier-Porte l'a relevé pour tout l'ouvrage de Pétis de La Croix, les questions morales se posent également ici, non sans ironie, dès les premières pages ${ }^{4}$. D'abord dans les conseils de la mère mourante

I. Fr. Pétis de La Croix, Les Mille et Un Jours, texte établi par P. Sebag, Paris, Christian Bourgois, I980. Noté ci-après Sebag. Voir aussi l'édition de référence de Raymonde Robert et al., qui réunit l'Histoire de la sultane de Perse et des vizirs, Les Mille et Un Jours et Les Aventures d'Abdalla, Paris, Honoré Champion, coll. "Bibliothèque des Génies et des Fées», $n^{\circ} 8$, Sources classiques n ${ }^{\circ} 54$, 2006.

2. Sebag, p. 49I-508

3. Sebag, p. 506

4. Ch. Bahier-Porte, «La mise en recueil des Mille et Un Jours», Féeries, n I, «Le Recueil», 2004, p. 93-I06, et spécialement p. 93. 
à ses filles : «Persévérez toujours dans la vertu que je vous ai enseignée, et suivez exactement les préceptes de notre grand prophète Mahomet ${ }^{5}$.» Puis dans l'attitude du premier vieillard tentant de convaincre les jeunes filles désargentées, désormais livrées à elles-mêmes, de se mettre sous sa protection : «Étant sans appui comme vous l'êtes, croyez-vous pouvoir éviter tous les pièges que le vice et la ruse ne manqueront pas de tendre à votre innocence ${ }^{6}$ ? Le vice et la vertu peuvent être pareillement soulignés, et même se superposer, mais sans viser particulièrement à faire rire, lorsqu'ils sont évoqués dans les récits comparables des Mille et Une Nuits par exemple. Le moment critique est souvent semblable. Dans le recueil arabe, l'oscillation entre bien et mal a lieu effectivement quand apparait l'orphelin, après la mort du père, quand les fils de marchand (plutôt que les filles de paysanne), oublieux des recommandations qui leur ont été faites, s'abandonnent à la débauche et dilapident l'héritage familial ... mais ils finissent par s'apercevoir de la fausseté des amis et par s'assagir, ou bien par trouver un père de substitution qui les remet en selle ${ }^{7}$. D'un autre côté, nous pouvons également rencontrer des narrations religieuses de "tentation", dont les formidables oscillations et les tempêtes cérébrales représentent la matière même $^{8}$. Dans quelle catégorie faudrait-il ranger Adis et Dahy? Il y a une moralisation indéniable du texte français, en dépit $\mathrm{du}$ ton ironique ou moqueur, dans sa couverture figurative, à travers de longs débats, probablement surajoutés, qui alourdissent l'événementiel. Mais l'action, elle non plus, n'est pas neutre, comme nous allons nous en rendre compte à travers ce bref résumé, et en commençant par le récitcadre d'Adis et Dahy (jours 194-210) :

Un jour, le calife Hârûn al-Rashîd et sa favorite, Sultanum, virent un vieillard et un jeune homme qui riaient fort. Curieux, le calife les fit venir

5. Sebag, p. 408.

6. Sebag, p. 4IO.

7. U. Marzolph et R. van Leeuwen, Arabian Nights Encyclopedia, 2 vol., ABC-CLIO, Santa Barbara, 2004, contes $\mathrm{n}^{\circ} 157,259,263$, 428, etc. Noté ci-après ANE. Le motif correspondant, Wi3r.r. Profligate, est répertorié chez H. M. El-Shamy, A Motif Index of The Thousand and One Nights, Indiana University Press, 2006, p. 3II. L'une des histoires de ce genre chez Galland est par exemple celle du Dormeur éveillé, voir A. Galland, Les Mille et Une Nuits, J.-P. Sermain (éd.), 3 vol., Paris, Garnier-Flammarion, 2004, vol. II, p. 425. Noté ci-après Galland.

8. D'une certaine manière, l'ensemble du Livre des Repentants ou Kitâb al-tawwâbîn d'Ibn Qudâma al-Maqdîsî (XII ${ }^{e}$-XIII ${ }^{\mathrm{e}}$ s.), G. Makdisi (éd.) (Damas, I96I), traite ce thème. Une autre histoire très célèbre dans le domaine arabe est celle du moine Barsisa qui cède à la tentation, histoire entrée en France (Histoire du santon Barsisa) presque en même temps que les Nuits grâce à l'ouvrage de 1707 de Pétis de La Croix, dans son Histoire de la sultane de Perse et des vizirs (voir ci-dessus note I). 
pour en connaître la raison. Ils riaient des histoires que l'un et l'autre venaient de se raconter et, à la demande du calife, le vieillard raconta la sienne :

Une paysanne très pauvre, qui avait deux filles, Fatime âgée de dixsept ans et Cadige de douze ans, mourut mordue par un serpent, laissant ses filles seules et démunies. Sur le chemin pour rapporter du linge en ville, les deux orphelines rencontrèrent un riche vieillard, Dahy, qui tomba amoureux de Cadige et proposa de les prendre toutes les deux chez lui. Cadige refusa, mais sur l'insistance de sa sœur et la promesse du vieillard de ne pas la toucher, elle finit par accepter. Cadige resta donc avec le vieillard, tandis que Fatime se rendit en ville pour rapporter le linge, mais elle ne revint plus et disparut. Toutes les recherches pour la retrouver échouèrent.

Trois mois plus tard, Cadige vit en rêve un bel homme qui se présenta comme celui qu'elle devrait un jour épouser, il lui reprocha d'avoir oublié Fatime et lui enjoignit de se rendre à l'île de Sumatra pour y retrouver sa sœur. Cadige et le vieillard Dahy prirent la mer pour Sumatra. Sur le bateau, Dahy raconta à Cadige sa véritable histoire : "Je suis un immortel, un génie, lui dit-il ; avec mon frère jumeau, Adis, nous avions pour charge de veiller sur Farzana, l'épouse de notre maître, le brahmane Cansou. Comme nous étions fort beaux, elle finit par nous séduire l'un et l'autre malgré notre résistance. Et comme elle se refusa à Torgut, un esclave noir, celui-ci, pour se venger, nous dénonça à notre maître qui, un jour, nous surprit tous les trois, son épouse, mon frère et moi nus dans le bain. Il punit son épouse ainsi que l'esclave, dont il avait appris les vraies motivations, en les métamorphosant en grenouilles. Quant à mon frère et moi, il nous condamna à vivre séparés sur terre comme des vieillards et de ne retrouver nos pouvoirs et notre apparence première que lorsque des jeunes filles de moins de vingt ans tomberaient amoureuses de nous. Deux cents ans se sont écoulés et nous n'avons toujours pas trouvé, ni l'un ni l'autre, de telles jeunes filles.» Cadige, touchée par son récit, avoua néanmoins qu'il lui était impossible de l'aimer sous cette apparence de vieillard. Ils poursuivirent leur navigation quand une tempête leur fit changer de route et aborder une ville inconnue.

Les habitants de cette ville préféraient précisément les vieillards aux jeunes gens. Dahy refusa cependant les avances de la reine du pays et fut jeté en prison. Il y retrouva son frère Adis. Ce dernier avait vu en rêve une villageoise de dix-huit ans (Fatime) qui l'avait invité à se rendre à l'île de Sumatra, son bateau fut détourné, il se retrouva lui aussi dans cette ville, puis en prison, car il avait également refusé de se laisser séduire. 
Les deux vieillards étaient tellement vieux qu'on les sortit de prison et qu'on les installa, malgré leur refus, sur l'ordre de la reine, dans une pagode pour les adorer. Là, ils se transformèrent subitement en deux beaux jeunes hommes, car il devait se trouver parmi la foule des adorateurs des jeunes filles de moins de vingt ans. À ce moment, leur maittre, le brahmane Cansou, apparut avec Fatime et leur expliqua qu'il avait lui-même enlevé Fatime et tout ourdi afin de les réunir à cet endroit. Dahy retrouva Cadige qui reconnut en lui le jeune homme de son rêve, Adis reconnut en Fatime la fille de son rêve. Le brahmane leur accorda la liberté et les deux couples partirent vivre ensemble dans une île habitée par les génies.» Le vieillard expliqua alors au calife Hârûn al-Rashîd que c'était là l'histoire qu'il avait racontée au jeune homme et qui justifiait leur hilarité9. Satisfait, le calife demanda alors au jeune homme de raconter à son tour son histoire (s'ensuit ici l'Histoire de Nasiraddolé et, à la fin de celle-ci, une autre histoire, Repsima, narrée également par le vieillard ["qui aimait beaucoup parler ${ }^{10}$ »], puis une autre encore, l'Histoire des deux pigeons, racontée par la favorite du calife).

Le noyau dur de l'Histoire des deux frères génies Adis et Dahy correspond globalement à une déchéance puis une réhabilitation de deux créatures immortelles. Au début chronologique du récit, quelque deux cents ans avant la naissance même des deux jeunes filles, les événements les plus importants ont lieu à l'écart du monde ordinaire des hommes. L'instance qui juge les deux génies immortels est un brahmane d'une longévité tout aussi remarquable et d'une puissance absolue, ses pouvoirs semblent équivalents à ceux d'un dieu. L'histoire se déroule donc selon un schéma à deux termes où des personnages (les deux génies) sont soumis à une autorité (le brahmane) qui veille à faire respecter l'ordre, avec un syntagme narratif très classique du type : démérite (des génies succombent aux charmes d'une femme) ; châtiment (le brahmane métamorphose les génies en vieillards et les exile sur terre). Le couple négatif (démérite, suivi de châtiment) et son symétrique positif (mérite, suivi de récompense) découlent d'une lecture essentiellement attentive à l'aspect éthique de l'action, une lecture où l'action est appréciée en termes de bien/mal. Les deux syntagmes (mérite/récompense; démérite/châtiment) forment le socle du récit moralisateur en général, et du religieux en particulier ${ }^{\mathrm{II}}$. C'est en ce sens que l'on

9. Sebag, p. 408-437.

Io. Sebag, p. 450.

II. Un des champs les plus féconds du récit religieux «moralisants» en Occident, qui partage souvent sa matière avec l'Orient, est représenté par le corpus des exempla médiévaux, voir Cl. Bremond, J. Le Goff et J.-Cl. Schmitt, "L'exemplum», dans Typologie des sources du Moyen Âge occidental, fasc. 40, Turnhout, Brepols, 1982 (rééd. 1996); voir aussi Cl. Bremond, «Les bons 
peut imaginer aisément que ce récit puisse être rattaché indifféremment au christianisme ou à l'islam, qui émanent d'ailleurs tous les deux des mêmes traditions orientales et sont des variations sur un même héritage.

Dans le cas d'Adis et Dahy, le syntagme narratif en question, si on entre davantage dans le détail, est encore plus complexe. Il introduit une clause suspensive, une condition, le châtiment n'étant pas définitif mais temporaire : il sera suspendu quand se sera réalisé un certain événement particulier, en l'occurrence obtenir l'amour d'une jeune fille, comme s'il fallait guérir l'adultère par le mariage. D'où l'enchaînement plus long mais plus précis, qui permet de lier le sort d'un immortel à une mortelle : démérite; châtiment conditionnel; réalisation de la condition (des jeunes filles tombent amoureuses des vieillards); fin du châtiment (les génies retrouvent leur apparence première et leur liberté). À partir de là, trois catégories d'histoires peuvent être dégagées : une catégorie proche du point de vue thématique, qui utilise la même couverture figurative, c'est-à-dire des personnages aux statuts analogues, avec des enjeux partagés entre le divin et l'humain, sur le thème de "l'ange déchu»; une catégorie plus proche du point de vue structurel, qui se développe selon le syntagme du «châtiment conditionnel» et ménage la réhabilitation du personnage déméritant quel que soit son statut; enfin, une troisième catégorie, à l'intersection des deux premières, qui possède à la fois les caractéristiques de l'une et de l'autre, représentée par une version particulière d'Adis et Dahy et qui peut aider à en comprendre la composition. La suite de ce travail sera donc articulée autour de ces trois catégories.

\section{Les anges déchus}

Appartiennent à la première catégorie les deux génies parmi les plus célèbres de l'islam, Hârût et Mârût, personnages coraniques appartenant à l'angélologie (II, IO2), abondamment commentés dans l'exégèse, immortels comme Adis et Dahy, déchus eux aussi pour avoir succombé aux charmes d'une femme. Seulement, leur châtiment n'a été assorti d'aucune condition suspensive. Ayant choisi d'expier leur faute sur terre, leur peine doit naturellement prendre fin avec la fin du monde ${ }^{\mathrm{I} 2}$. Nous avons dans Hârût

récompensés et les méchants punis", dans $\mathrm{Cl}$. Chabrol (éd.), Sémiotique narrative et textuelle, Paris, Larousse, I973, p. 96-I21.

I2. Voir l'excellent article d'Alireza Shapour Shahbazi, «Hârut and Mârut", dans Encyclopaedia Iranica, vol. XII, fasc. I, 2003, p. 20-22; sur le thème de l'ange déchu, il est encore intéressant de citer l'article de Leo Jung, "Fallen Angels in Jewish, Christian and Mohammedan Literature, A Study in Comparative Folk-Lore", dans The Jewish Quarterly Review, vol. 15, n 4, 1925, p. 467-502. 
et Mârût les mêmes éléments que dans Adis et Dahy mais un traitement différent. Si dans les divers textes arabes le discours est résolument religieux et l'amour définitivement banni de la vie des deux anges, en revanche, dans Les Mille et Un Jours, le ton est badin, humoristique, parfois même grotesque. Il convient à ce propos de reprendre le récit-cadre d'Adis et Dahy, qui montre bien une certaine forme de désinvolture ou d'incompréhension de l'orientaliste, ou plus probablement de son collaborateur Lesage, vis-à-vis de l'Orient ${ }^{\mathrm{I} 3}$.

Dans Les Mille et Un Jours, Hârûn al-Rashîd, le calife abbasside devenu célèbre grâce aux Mille et Une Nuits, voit son attention attirée par deux hommes "parce qu'ils riaient à gorge déployée. Comme il était naturellement curieux $[\ldots]^{14} »$. C'est ainsi que le calife demandera à écouter successivement plusieurs histoires, dont celle d'Adis et Dahy, seulement pour satisfaire sa curiosité, parce que ces histoires font rire. À la fin, il donnera une récompense aux conteurs. Un tel comportement est-il habituel de la part d'un homme qui règne sur l'un des premiers et plus puissants empires de son époque? La réponse dépend du point de vue. Il est vrai que l'on peut lire dans la traduction d'Antoine Galland des Nuits, au début de l'Histoire de trois calenders, que le calife, qui voit une jeune femme battre violemment deux chiennes puis pleurer avec elles de leur douleur, ne peut réprimer sa curiosité et cherchera à en connaître la cause. Il est vrai également que ce même calife, lorsqu'il voit dans la rue un homme «maltraiter cruellement» une jument (Histoire de Sidi Nouman) ou un mendiant demander à ce qu'on lui donne un soufflet lorsqu'on lui fait l'aumône (Histoire de l'aveugle Baba-Abdalla), convoque tous ces gens pour leur demander des explications et donc pour entendre leur histoire ${ }^{15}$. Seulement, derrière l'histoire, il y a un désordre à réparer. Le calife, à chaque fois, fera cesser les violences, les animaux ou les hommes cesseront d'être maltraités, et, s'il y a eu injustice ou abus, le calife rétablira les victimes dans leur droit.

Sur le plan narratif, dans les Nuits, lorsque le calife Hârûn al-Rashîd est présent, l'histoire encadrée se poursuit en quelque sorte après sa narration dans le récit qui l'encadre et avec lequel elle va se confondre (ce qui est

I3. Ch. Bahier-Porte, «Le laboratoire des Mille et Un Jours : de François Pétis de La Croix et Lesage", dans R. Jomand-Baudry et J.-Fr. Perrin (éds), dans Le conte merveilleux au XVIII siècle, Une poétique expérimentale, Paris, Kimé, 2002, p. 23-40, et 23-24: "Nous sommes pour notre part convaincue de la participation de Lesage au remaniement des manuscrits traduits ou annotés par l'orientaliste."

I4. Sebag, p. 408.

I5. Galland, vol. I, p. I3I et vol. III, p. I2O-I22. 
rarement le cas par exemple des récits rapportés par Shahrazâd). Le calife, lui, intervient dans l'histoire d'autrui, et d'auditeur il se transforme en acteur, il change le cours des événements et agit de sorte à créer un autre dénouement. Il dénoue l'intrigue. Le rôle du calife, auditeur, acteur, justicier, réparateur des désordres, Galland l'a bien compris. Même dans l'Histoire de Cogia Hassan Alhabbal, qui a été plus ou moins artificiellement ajoutée au cycle des Aventures du calife Haroun-al-Raschid, comme on peut en voir les traces dans le Journal de Galland (Io mai I709) ${ }^{16}$, le calife ne se contente pas d'écouter l'histoire et de congédier à la fin le narrateur, il la poursuit : "Le diamant qui a fait ta fortune est dans mon trésor.» Il lui enjoint ensuite de faire venir Saadi et, surtout, Saad afin "qu'il reconnaisse que l'argent n'est pas toujours un moyen $[\ldots]^{17}$ ». Grâce au calife, et dans ce cas précis à l'intelligence de Galland, l'aventure de Cogia Hassan Alhabbal se prolonge encore dans le récit qui l'encadre, afin de lever les dernières incertitudes et que la vérité (la morale) puisse s'imposer. Autrement dit, le calife n'est pas un amateur effacé de récits amusants, c'est un juge recherché (voir l'Histoire du faux calife ${ }^{18}$ ) et redouté. Et audelà de la curiosité, ingrédient que l'on fait miroiter aux lecteurs, il y a au fond, pour le calife de Bagdad, l'obligation légale de faire régner la paix et la justice. En islam, en effet, tout crime resté impuni, tout désordre, toute injustice, qui ont lieu durant le règne de tel calife, engagent la responsabilité de ce calife. Il en sera comptable devant Dieu ${ }^{19}$. La fonction califale est une fonction à risque, qui n'a pas toujours été prise au sérieux sans doute par l'un des adaptateurs des Mille et Un Jours. Elle a été réduite à l'exercice d'un pouvoir qui interpelle des passants pour leur ordonner de raconter

I6. BnF, ms nº ${ }^{\circ} 277$, p. 93, vu le Ir février 2016 : http://gallica.bnf.fr/ark:/I2I48/btvib9o6I484b/ f65.image

17. Galland, vol. III, p. I79.

18. ANE, $\mathrm{n}^{\circ} 73$.

19. Les Mille et Une Nuits elles aussi en rendent compte, comme dans Les trois pommes, lorsque le calife s'écrie (à l'adresse de son vizir) à la découverte de la dame assassinée (Galland, vol. I, p. 293) : «[...] On commet impunément sous ton ministère des assassinats dans ma capitale, et l'on jette mes sujets dans le Tigre, afin qu'ils crient vengeance contre moi au jour du jugement. " Par comparaison avec le texte arabe (M. Mahdi, Alf layla wa-layla, Leyde, Brill, 1984, p. 220, noté ciaprès Mahdi), Galland, d'une part, omet de préciser que le calife "s'affligea et se mit à pleurer à ce spectacle» et, d'autre part, transforme dans sa traduction «dans ma ville» par «sous ton ministère», pour placer le meurtre sous la responsabilité de son vizir, ce qui n'était pas dans les intentions du texte arabe. La traduction de Bencheikh et Miquel (Les Mille et Une Nuits, Paris, Gallimard, coll. «Bibliothèque de la Pléiade», 2005, t. I, p. I5I, noté ci-après Bancheikh et Miquel), même si elle a été faite sur une autre version des Nuits, est très claire à cet égard : "Chien de vizir, est-ce ainsi qu'on tue les gens sous mon règne, qu'on les jette au fleuve et qu'on me laisse responsable du sort qui leur est fait?» Ce genre de méfait engage en effet la responsabilité du calife ici-bas et dans l'au-delà. 
leur histoire sans chercher à s'en faire le juge, ni à en tirer un autre profit (par exemple de la faire enregistrer par écrit pour les générations futures) que celui de satisfaire sa curiosité. Le calife de Pétis de La Croix ou de Lesage prend les traits d'un personnage passif, davantage soucieux de son bon plaisir que de son peuple.

La posture générale est en effet la même dans l'ensemble d'Adis et Dahy: faire rire. À entendre l'histoire, rappelons-le, le couple de promeneurs rit «à gorge déployée». C'est ainsi du moins que l'on désire modéliser l'attitude du lecteur de ce conte des Mille et Un Jours. Le drame des anges déchus, Hârût et Mârût, qui ont été enfermés jusqu’à la fin des temps et dont la maîtresse a été transformée en (planète) Vénus, ce drame fondateur fait donc place à la comédie des deux génies Adis et Dahy, dont la première maitresse a été transformée en grenouille (métamorphose caricaturale), et qui sont désormais des vieillards centenaires amoureux de deux jolies paysannes. L'Orient apparaît comme un univers propice, sans davantage d'explications, à l'insertion de mœurs, certes fort respectueuses de la morale, mais cocasses et un peu ridicules. L'exemple le plus frappant à cet égard est l'île où les vieillards seront délivrés, plus précisément la description des habitants de l'île et leur comportement : "Leur habit n'était pas moins singulier que leurs manières. Ils avaient de longues robes de toile de coton, où l'on voyait peintes en rouge, vert et jaune, diverses figures de démons, avec des flammes et d'autres grotesques, et ils portaient à la tête un long chapeau pointu fait de carton, et enduit aussi de différentes couleurs ${ }^{20}$.» En outre, les habitants ont horreur de la jeunesse, qu'ils voient comme une laideur, et tiennent les vieux pour les plus beaux. Les deux vieillards, Adis et Dahy, seront alors aimés, verront du coup finir leur châtiment, retrouveront leur forme première et leur liberté. La délivrance est venue du grotesque.

\section{Le châtiment conditionnel}

Le motif du châtiment qui cessera quand seront réalisées certaines conditions est assez fréquent dans les contes du folklore. L'une de ses manifestations les plus célèbres est celle de la jeune femme métamorphosée ou condamnée à un sommeil proche de la mort, qui reprendra vie ou qui retrouvera son apparence humaine à la réalisation d'une certaine condi-

20. Sebag, p. 429. 
tion (AT 409A, The Girl as Goat; AT 4IO, Sleeping Beauty) ${ }^{21}$. L'exemple que nous allons voir maintenant est extrait du Trône enchanté, texte traduit du persan en I8I7 par Daniel Lescalier (I743-I822), dit aussi le Baron Lescallier (Imprimerie de J. Desnoues, New York). Il possède en gros les mêmes origines que les sources utilisées par Pétis de La Croix pour ses Mille et Un Jours. On peut le décomposer en deux parties. La première partie, que l'on peut appeler «Le brahmane capable de décrire l'épouse du roi sans l'avoir vue", fonctionne comme un récit-cadre de la seconde partie et connaît des équivalents dans la littérature arabe classique, comme d'ailleurs dans les Mille et Une Nuits. La seconde partie, à la fois récit enchâssé et récit enchâssant, que l'on peut appeler "Le prince fou à qui l'on doit raconter sa propre histoire pour le guérir", suit la même structure qu'Adis et Dahy et offre en outre un intérêt tout à fait remarquable pour une compréhension plus fine de nombreux récit-cadres, dont celui des Mille et Une Nuits.

\section{Le brahmane capable de décrire l'épouse du roi sans l'avoir vue}

Un roi ne pouvait quitter des yeux son épouse tant il l'aimait. Sur le conseil de son vizir, il fit faire le portrait de la jeune femme pour que son image puisse l'accompagner partout plus commodément. Le roi demanda cependant à un religieux, un brahmane qui possédait "de rares connaissances dans l'art cabalistique, la magie et les sciences occultes ${ }^{22}$ » si le portrait lui semblait exact. Le brahmane prit un pinceau et laissa tomber une goutte d'encre. Sur la peinture, l'encre fit comme un grain de beauté sous le sein de la jeune femme. Le brahmane estima que le portrait était désormais parfait. Le roi, persuadé que le brahmane devait connaître intimement la reine, donna l'ordre au vizir de le mettre à mort. Le vizir emmena le brahmane et le cacha, se disant qu'un tel jugement pris dans la précipitation pouvait être injuste et que le religieux pourrait se révéler fort utile à l'avenir. En effet, le prince étant devenu fou, personne ne réussit à le guérir, sauf le brahmane, que l'on avait fait passer pour une jeune fille, qui devina exactement son mal et lui rendit la raison. Le roi, très étonné par sa science, demanda comment on avait pu deviner le mal de son fils. «De la même façon que le brahmane avait pu deviner le portrait de la reine», s'entendit-il répondre. Le roi comprit qu'il avait mal agi envers le brah-

2I. A. Aarne et S. Thompson, The Types of the Folktale, Helsinki Academia Scientiarum Fennica, I964.

22. Le trône enchanté, trad. Le Baron Lescallier, Imprimerie de J. Desnoues, New-York, I817, p. 68. Noté ci-après Trône. 
mane, celui-ci se montra alors à lui, il fut pardonné, récompensé et reprit sa place auprès du roi ${ }^{23}$.

\section{Le prince fou à qui l'on doit raconter sa propre histoire pour le guérir}

Un jour, le fils du roi partit seul à la chasse et s'arrêta pour boire. Il fut surpris par des lions et dut se réfugier dans un arbre où se trouvait déjà un gros singe. Celui-ci rassura le prince et promit de le protéger. La nuit, le singe proposa au prince de dormir pendant que lui veillait. Une fois le prince endormi, le lion essaya de convaincre le singe de lui jeter le prince, mais, fidèle à sa parole, le singe refusa. Plus tard dans la nuit, le prince se réveilla et proposa au singe de dormir, lui promettant à son tour de le protéger. Une fois le singe endormi, le lion réussit à convaincre le prince de lui jeter le singe, le prince s'apprêtait à faire tomber le singe, lorsque ce dernier se réveilla, comprit que le prince l'avait trahi, et, malgré tout, lui pardonna. Le prince, honteux, demanda au singe un moyen pour expier sa faute. Lui versant un peu d'urine dans son oreille, le singe rendit fou le prince : il retrouvera la raison lorsqu'on lui racontera point par point son histoire avec le lion et le singe et comment il avait trahi ce dernier et était devenu fou. Le roi finit par retrouver son fils, mais celui-ci avait un comportement insensé. Aucun médecin ne parvint à le guérir. Le vizir fit appel au sage brahmane qui, quatre jours de suite, fit venir le roi et le prince malade et leur raconta chaque jour une partie de l'histoire du prince, depuis son départ à la chasse jusqu'à ce qu'il devienne fou. Au quatrième jour, après avoir écouté son histoire du début jusqu'à la fin, le prince retrouva la raison. Le brahmane, que le roi avait voulu mettre à mort, fut pardonné et récompensé 24 .

Ces deux morceaux, qui ont été réunis en une même histoire, correspondent à deux traditions narratives différentes et extrêmement intéressantes l'une et l'autre. Commençons par la première, qui est un peu en dehors de notre sujet ici, mais qui poursuit en quelque sorte un ancien travail de recherche publié en 1998, auquel elle apporte des arguments supplémentaires ${ }^{25}$. Il s'agit, très brièvement, du rôle premier du poète dans l'Arabie préislamique, rôle que j'ai voulu montrer comme équivalent à celui d'un homme de religion, en milieu païen, et qui s'est poursuivi

23. Trône, p. 66-86.

24. Trône, p. 7I-85.

25. "Classification des traditions narratives arabes par conte-type : application à l'étude de quelques rôles de poète", Bulletin d'Études Orientales, Damas, IFEAD, I998, t. L, p. 29-59. 
en se dissipant progressivement, longtemps après l'arrivée de l'islam. On en trouve les traces dans divers biographies de poètes célèbres, agissant comme des "devins", capables notamment de faire une description très précise d'une femme qu'ils n'ont pourtant jamais vue, et qui s'attirent ainsi les soupçons et la colère du mari. L'idée est que ce rôle est d'abord celui d'un prophète (le prophète de l'islam a précisément été traité de poète à ses débuts : Coran, LXIX, 4I). Nous en trouvons une version amusante dans les Mille et Une Nuits, dans la traduction de Bencheikh et Miquel : le poète Abû Nuwâs parvient à décrire au calife Hârûn alRashîd avec une extraordinaire exactitude son épouse Zubayda en train de prendre son bain ${ }^{26}$. Le calife est d'abord persuadé que le poète a dû voir la scène, et veut le mettre à mort (comme le roi veut mettre à mort le brahmane), puis il comprend que c'est son pouvoir de poète qui lui permet de telles prouesses et l'épargne. Le ton est humoristique mais la fonction du poète, détenteur de pouvoir magique de divination, comme précisément le brahmane qui a le statut d'homme de religion (et c'est en cela qu'il est précieux), transparait bien derrière le divertissement. En outre, le fait qu'il s'agisse précisément d'un divertissement permet de lever toute censure, de disqualifier le poète (vision d'une femme dans son bain) réduit au rôle de bouffon et dépossédé du sérieux et de l'amplitude d'un prophète (vision du destin de l'humanité, de l'univers, de la création).

Le deuxième morceau est également une clé pour la lecture et l'interprétation d'autres textes. D'abord, par rapport à Adis et Dahy, il faut bien constater une remarquable proximité sur le plan structurel : démérite (infidélité conjugale, trahison de l'amitié); châtiment (vieillesse ou perte de la jeunesse, folie ou perte de la raison); condition de la levée du châtiment (obtenir l'amour d'une jeune femme, écouter raconter sa propre histoire). Si la structure est identique, la différence la plus significative entre les deux histoires, même si des recettes similaires ont été appliquées, semble se situer au niveau de la condition de la levée du châtiment. Celle du jeune prince est remarquable. Par rapport à de nombreux autres ouvrages, Décameron, Sept vizirs ou Sept sages de Rome, Mille et Une Nuits, Cent et Une Nuits, Kalîla et Dima, Contes du vampire, Contes du perroquet, etc., rares sont les enchâssements aussi clairement motivés. Dans Le Prince fou à qui l'on doit raconter sa propre histoire pour le guérir, son histoire est narrée une seconde fois à l'intérieur d'elle-même. L'enchâssement est réalisé à l'iden-

26. Bencheikh et Miquel, II, p. I49, Conte de Zubayda au bain; ou bien encore sur le même thème, Bencheikh et Miquel, II, p. 34, Conte du calife, d'Abû Nuwâs et de la jeune esclave; voir ANE, $n^{\circ} 85$ et $n^{\circ}$ III. 
tique parce qu'il est indispensable au dénouement. Il est le dénouement, en ce sens qu'il libère définitivement le prince. Les conditions sont particulières : l'auditeur principal est blessé dans son âme, malade, traumatisé par son méfait : «La crainte et la honte ôtaient au prince le courage de lever les yeux ${ }^{27}$.» Nous sommes à l'opposé, par exemple, du calife Hârûn al-Rashîd, dans le récit-cadre de Adis et Dahy, où il représentait un auditeur en parfaite santé ou dans le cas de Galland, où le calife est surtout attentif à l'ordre public. Mais même pour le calife Hârûn al-Rashîd, il existe des histoires où il est souffrant (insomnie) et où, après avoir entendu raconter une histoire, il guérit : «Le cœur du calife retrouva sa sérénité et l'insomnie dont il souffrait disparut ${ }^{28}$.»Cela, c'est l'effet thérapeutique des récits en général, même lorsque l'histoire racontée n'a aucun rapport avec la vie ou la situation de celui qui l'écoute.

Dans les Mille et Une Nuits, dans le récit-cadre, longtemps il a été question, parmi les chercheurs, de la maladie et de la folie du roi Shahriyâr, celui qui, traumatisé par la trahison de son épouse (un motif banal), décide de prendre chaque jour une nouvelle épouse et de la mettre à mort le lendemain (une sorte de folie meurtrière) ${ }^{29}$. Le texte arabe n'en est pas dupe. Il fait dire à Shahriyâr, lorsque ce dernier apprend que son jeune frère a été trompé par son épouse, et de manière bien plus précise que Galland : «Par Dieu, si cela m’était arrivé, j'aurais tué au moins cent femmes ou mille femmes et j'en aurais perdu l'esprit, je serais devenu fou ${ }^{30}$." Et la suite confirmera les prévisions du monarque, qui deviendra fou. Jusqu’à

27. Trône, p. 76.

28. Bencheikh et Miquel, II, p. I9, conclusion du Conte des amours de Budîr et Jubayr; ANE, $\mathrm{n}^{\circ}$ 83.

29. Voir, par exemple, ANE, $\mathrm{n}^{\circ} \mathrm{I}, \mathrm{p} .374$ : "The story's most general interpretation implies that Shahriyâr has lost his psychic balance by witnessing his wife's illicit sexual relations; he takes refuge in a highly compulsive way of behaving, in order to reestablish his mental equilibrium. By telling him stories, Shahrazâd manages to cure him of the trauma he experienced, restoring to him a balanced perspective on life"; H. Mehio, "Shahriyâr est-il un tueur en série?", dans A. Chraïbi (éd.), Les Mille et Une Nuits en partage, Arles, Actes Sud, 2004, p.70-80; J. Zipes, When Dreams Came True: Classical Fairy Tales and Their Tradition, New York, Routledge, 2007, p. 6o.

30. Mahdi, p. 6I, pour "perdre l'esprit» et «folie» l'arabe utilise la même racine j-n-n : «kunt atajannan wa-akhruj majnûn». La traduction de Hadawi (The Arabian Nights, New York, W. W. Norton \& Co., I990, p. 9), qui n'est pas très précise ici, semble suivre dans un premier temps Galland avant de reprendre ce que le texte arabe dit clairement : "I would have killed at least a hundred or even a thousand women. I would have been furious (sic); I would have gone mad." Galland, que l'on voit bien suivre ici la leçon de ce manuscrit, simplifie la phrase et omet de traduire la folie annoncée du roi, se contentant de la «rage" (qui désigne en réalité la folie), p. 29 : "Je crois que j'en aurais sacrifié plus de mille à ma rage (sic) »; ce qui est dommageable à une bonne interprétation, pendant des siècles en Europe, de l'interprétation, par les lecteurs de langue arabe, du comportement du roi. 
l'arrivée de Shahrazâd dont les récits semblent apaiser momentanément Shahriyâr, même s'il a été reproché à la conteuse, notamment par Pétis de La Croix dans sa préface des Mille et Un Jours, de ne pas faire correctement son travail ${ }^{3 \mathrm{I}}$ : car comment compte-t-elle changer la mauvaise opinion du roi Shahriyâr, son auditeur, à propos des femmes si elle lui rapporte des histoires où les épouses sont encore et toujours infidèles (Premier vieillard, Troisième vieillard, Roi des îles noires, ...)? Mais le but est-il réellement de changer l'opinion du roi ou simplement de lui montrer la banalité de ce qu'il a subi (un cocuage) et la disproportion de sa réaction (le meurtre à grande échelle) ${ }^{32}$ ? Cela, le texte arabe, après l'avoir signifié implicitement, en y ajoutant le thème de l'injustice des puissants de ce monde et de la punition disproportionnée, le texte arabe donc va le dire explicitement, non pas dans la version de Galland mais dans des manuscrits plus complets antérieurs à sa traduction, toujours par la voix de Shahrazâd : "Cette affaire qui t'est arrivée, il en est arrivé de plus grave encore aux rois qui t'ont précédé33.» Autrement dit, le roi est davantage victime de lui-même et de son ignorance du monde que de la trahison de son épouse. Mieux encore, la perspicace Shahrazâd va agir exactement comme pour Le Prince fou à qui l'on doit raconter sa propre histoire pour le guérir. Elle fait raconter par l'un des préfets du sultan Baybars un résumé du récit-cadre des Nuits $^{34}$. Nous y retrouvons tous ce qui est arrivé aux deux rois, la décision de l'aînée de prendre chaque jour une femme et de la faire exécuter le lendemain, jusqu'à l'apparition de Shahrazâd et l'entrée dans la routine de la narration par nuitée. Des traces (le retour du jeune roi parce qu'il veut embrasser une dernière fois son épouse, et non pas à cause d'un oubli; la population qui se plaint à Dieu et non pas le vizir qui est en mauvaise passe) renvoient clairement vers un récit-cadre davantage littérarisé et islamisé du type de celui utilisé par Galland (texte de famille syrienne). Le plus intéressant dans le manuscrit Kayseri des Mille et Une Nuits est la réaction du roi : «Lorsque le roi Shahriyâr entendit cette histoire, il se ressaisit et retrouva la raison (littéralement, quitta l'ivresse où il se trouvait) et se dit : "Par Dieu, cette histoire est mon histoire et ce récit est mon récit, j'étais dans la peine et la tourmente jusqu'à ce que celle-ci (Shahrazâd) me remette sur le droit chemin. Loué soit Dieu qui est la

31. Sebag, p. Io.

32. On pourra consulter avec profit l'analyse faite par Jean-Paul Sermain, Les Mille et Une Nuits entre Orient et Occident, Paris, Desjonquères, 2009, p. 42.

33. Manuscrit Kayseri, $\mathrm{n}^{\circ} 674$, fol. I23b.

34. Manuscrit Kayseri, $n^{\circ}$ 674, fol. I2ob. 
cause de toutes les causes et le sauveteur de toutes les âmes." Puis il lui dit : "Ô Shahrazâd, tu m'as appris bien des choses utiles et tu m'as sauvé de mon ignorance." ${ }^{35}$." Miroir n'est pas un vain mot pour décrire l'opération de catharsis subie par le roi dans les Mille et Une Nuits afin de le guérir de sa folie, comme pour le prince devenu fou parce qu'il a trahi le singe. Le procédé est parfaitement maitrisé dans les textes anciens.

Très intéressante aussi, sur le plan éthique, la double perspective qui nous a été offerte à travers ces deux variantes : point de vue de la victime d'une trahison (le roi Shahriyâr victime de son épouse infidèle) et point de vue du coupable d'une trahison (le prince coupable envers le singe). La double perspective permet d'apprécier l'incertitude ou l'ambiguïté qui entoure, toujours sur le plan de l'appréciation éthique, le comportement des personnages. Voilà le roi Shahriyâr qui, de victime, va se transformer en coupable d'une série de meurtres, d'une folie tournée contre les autres et dont il faudra le soigner. De l'autre côté, le prince, de coupable, à cause de sa volonté d'expier sa faute, le voilà transformé en victime de ses remords et d'une folie tournée contre lui-même. Autrement dit, on va sauver une victime-coupable ou bien un coupable-victime. Lapproche morale dans ces récits utilise des grilles de lecture très fines, très attentives à l'instabilité de l'être humain et à sa complexité, que ce soit pour le racheter ou le prévenir contre la chute. Cependant, à voir de près, ce n'est pas la même morale qui est appliquée aux hommes et aux femmes, l'inégalité reste forte de ce point de vue, y compris dans Adis et Dahy. Les femmes coupables sont souvent sévèrement punies sans possibilités de rachat (l'enjeu pour les rois touche à la légitimité de la descendance et donc à la transmission du pouvoir) : exécutées dans les Nuits, métamorphosées en bêtes dans Adis et Dahy.

\section{L’ange déchu et le châtiment conditionnel}

La troisième et dernière partie de ce travail propose une histoire où le motif de l'ange déchu est inséré dans un enchaînement narratif de type "châtiment conditionnel ", c'est-à-dire les deux éléments que nous venons de voir et qui correspondent, réunis ensemble, à Adis et Dahy. Cette histoire se trouve également dans Le trône enchanté, c'est même la première histoire racontée. Elle est enchâssée dans un récit-cadre assez intéressant, car avec des protagonistes divins motivés par des objectifs moraux. Celle qui écoute l'histoire est en effet Parvati (ou Parbaty), l'épouse du dieu

35. Manuscrit Kayseri, $\mathrm{n}^{\circ}$ 674, fol. I23b. 
Shiva (Mahadiou), à qui elle demande de lui raconter «une histoire ou quelques aventures remarquables des Rajahs les plus renommés pour leurs vertus et pour avoir donné au monde des exemples de grandeur d'âme, de générosité et de bravoure au-dessus des autres» (p. I-2), des récits exemplaires donc... et c'est ainsi que le dieu Shiva va raconter à son épouse Parvati ce qui est peut-être à l'origine de Adis et Dahy et qui relève d'un récit à caractère mythologique fondateur ("Origines des Rajahs de Malouah ", selon l'intitulé même du texte) ${ }^{36}$. Le résumé :

\section{Canderbessine}

Un génie du nom de Canderbessine séduisit des nymphes célestes, des Apsaras, si bien que le grand génie Indra (Aïndra) décida de le métamorphoser en âne et de le bannir sur terre. Canderbessine ayant longuement supplié Indra, celui-ci ajouta que "Canderbessine sera âne le jour et reprendra sa forme naturelle la nuit, en outre, quand il aura un enfant avec une fille de Rajah et que celui-ci aura brûlé la peau d'âne, il sera définitivement libéré et pourra retourner auprès d'Indra». Tout se passa ainsi. Le génie Canderbessine parvint à épouser une princesse, grâce à la construction en une nuit d'un palais extraordinaire, et à lui donner un enfant, Békermadjiet (héros «historique» auquel sera consacré le reste du livre); il fit au passage un autre enfant à une autre jolie fille, sa peau d'âne fut brûlée par le père de la princesse. Il quitta alors la terre et remonta définitivement au ciel auprès d'Indra ${ }^{37}$.

Sur les plans structurels et thématiques, la proximité entre Canderbessine et Adis et Dahy est assez remarquable. Le démérite est le même (relation amoureuse interdite), la punition est conditionnelle dans les deux cas : c'est un exil terrestre sous une forme désavantageuse (vieillard, âne). La fin du châtiment est suspendue à une condition analogue (une liaison amoureuse légitime), avec des clauses supplémentaires dans le cas de Canderbessine, qui doit attendre d'avoir un fils et que sa peau d'âne soit brûlée pour être entièrement libre. Il y a également des différences dans la fin choisie, puisque Canderbessine abandonne femmes et enfants et remonte au ciel tandis qu'Adis et Dahy demeurent curieusement, eux des immortels, avec leur épouse mortelle. Certains éléments de Canderbessine rappellent les contes du folklore (la peau d'âne, le palais construit en une nuit), d'autres des récits mythologiques (l'enfant né d'un père d'origine

36. Trône, p. I

37. Trône, p. I-I4. 
céleste) ${ }^{38}$. En effet, toute l'histoire de Canderbessine est tournée vers l'obtention de cet enfant moitié homme moitié dieu (ou génie), qui aura un destin exceptionnel et dont les exploits vont remplir le reste de l'ouvrage. L'exigence morale (démérite premier de Canderbessine qui corrompt les nymphes célestes) semble n'être qu'un prétexte. La morale est faible, d'autant plus que le comportement de Canderbessine, une fois sur terre, n'a guère changé : incorrigible, il séduit une jolie suivante de la princesse et lui fait un enfant, en plus de celui qu'il va avoir avec son épouse légitime. Personne ne lui en tiendra rigueur, ni dans le ciel ni sur terre. En outre, Canderbessine ne fait preuve d'aucun mérite personnel pour obtenir la main de la princesse, son séjour est d'assez courte durée, la construction d'un palais en une nuit a été réalisée assez facilement, à sa demande, par un génie ami. Les conditions qui lui ont été imposées ont été levées sans peine. Comme si le texte nous provenait d'une époque où l'on ne demande pas aux Dieux exactement les mêmes choses qu'aux hommes, leur morale est plus lâche et leur semence la bienvenue pour féconder parmi les humains des êtres exceptionnels, comme Hercule fils de Zeus (d'ailleurs le fils de Canderbessine «sera doué d'une force égale à celle de cent éléphants ") ${ }^{39}$. Du coup, Adis et Dahy paraît plus ordinaire, plus "bourgeois", et à cause de cela finalement plus respectueux de la bonne morale, en dépit de tentatives ironiques ou moqueuses.

L'aspect répétitif de l'ensemble de ces récits, après examen, se dégage avec netteté. Une répétition structurellement indispensable au dénouement, non pas seulement celle de la " $2^{\mathrm{e}}$ chance», de la prise de distance et de l'apprentissage, mais aussi, d'une certaine manière, de la clôture du monde et de sa finitude. Elle semble correspondre à ce que disait le poète Abû Nuwâs (VIII ${ }^{\mathrm{e}}$-IX ${ }^{\mathrm{e}}$ siècle) lorsqu'on lui reprochait les maux provoqués par le vin : «Ne fais point de reproche, il est tentation / ce qui fut mon malheur sera ma guérison"; ou mieux encore, à ce qu’on peut lire dans

38. Pour la métamorphose en âne et la peau à détruire (à ne pas confondre avec la princesse qui se déguise sous une peau d'âne), voir par exemple le conte type AT 430, The Ass, et le motif très fréquent D72I.3. Disenchantment by distroying skin (covering). Pour la construction d'un palais en une nuit afin d'épouser la princesse, l'exemple le plus célèbre se trouve dans Aladdin, qui est luimême construit pour une part sur le conte type AT 560, The Magic Ring, et le motif Diızi.I. Castle produced by magic. Enfin, pour le mari non seulement surnaturel, mais d'origine divine et qui va donner naissance à une personnalité remarquable de l'histoire humaine, fondatrice d'une dynastie, d'un empire ou d'une religion, les cas abondent en Orient, comme par exemple Alexandre de Macédoine, voir Pseudo-Callisthène, Le roman d'Alexandre, trad. G. Bounoure et Bl. Serret, Paris, Les Belles Lettres, I992, p. 5 : «Olympias [...] voit le dieu Ammon, cette nuit même, l'enlacer [...].» Même s'il s'agit d'un artifice, l'imaginaire attaché à un tel acte est présent.

39. Trône, p. I4. 
le traité sur l'amour d'Ibn Hazm (xi ${ }^{\mathrm{e}}$ siècle), Le Collier de la colombe : "L'amour, que Dieu te garde, est un mal harassant qui porte en lui son propre remède ${ }^{40}$.» C'est en effet ce qui arrive aux personnages des histoires que nous venons de voir en commençant par Adis et Dahy. Les deux génies ont chu à cause de l'amour et de la femme, qui se superposent ici, et trouveront leur salut grâce à l'amour et à la femme. Le prince devient fou à cause de sa trahison et retrouve la raison à la revoir. La formule d'Ibn Hazm est également vraie pour le roi Shahriyâr, meurtri par une épouse adultère, sauvé par une épouse intelligente, dans le récit-cadre des Nuits, ou pour le génie Canderbessine, dans Le Trône enchanté, puni à cause de nymphes célestes et racheté par une princesse terrestre. La différence fondamentale avec Adis et Dahy est dans l'épisode additionnel moralisateur d'acquisition de mérite : les deux vieillards sont fortement sollicités par les femmes de l'île sur laquelle ils sont arrivés par accident, mais ils ne cèdent pas; ils préferent même être jetés en prison plutôt que de se laisser séduire par la reine et sa sœur. Eux qui n'ont pas su résister au début de l'histoire, les voilà capables de le faire à la fin. Ils ont changé, et ils restent par la suite attachés à vivre avec leur épouse.

En somme, une fin heureuse pour une famille ordinaire, alors qu'au départ il s'agissait tout de même de deux génies immortels aux pouvoirs extraordinaires. Pourquoi à la fin ce sous-emploi? Est-ce une sorte de composition métaphorique : derrière chaque vieillard, se cache son esprit, ou plus précisément son âme, belle, pleine de vigueur, immortelle, fidèle, digne de l'amour même des plus jeunes filles? Leur sous-emploi serait-il lié à une volonté de modération, peut-être inconsciente, par rapport à Canderbessine par exemple, qui les pousse à fuir tout excès et à se ranger, et qui aplatit le récit? Ou bien, comme pour Hârûn al-Rashîd, est-ce simplement une certaine désinvolture vis-à-vis d'une matière décomposée, recomposée, transformée, réécrite, rattachée à un Orient grotesque pour rire? Certains motifs importants du conte d'Adis et Dahy n'ont pas été abordés ici, comme celui du rêve (comme moyen de communication et d'exercice d'influence), de l'esclave noir qui ne peut séduire la reine (qui renvoie à l'esclave noir amant de la reine, dans les Nuits), ou encore celui de la fratrie (le même que dans le récit-cadre des Nuits: deux frères vs deux sœurs). Cependant, même en nous arrêtant à ce stade de l'analyse, la mul-

40. Ibn Qutayba, Introduction au livre de la poésie et des poètes, éd. et trad. M. Gaudefroy Demombynes, Paris, Les Belles Lettres, 1947, p. I2; Dîwân Abî Nuwâs, Ewald Wagner (éd.), Stuttgart, Franz Steiner, 1988, vol. 3, p. 2; Ibn Hazm, Tawq al-hamâma, Beyrouth, éd. Ihsân 'Abbâs, I993, p. I00. 
tiplicité des perspectives et des questions soulevées révèle tout l'intérêt d'une comparaison avec les sources arabes, turques ou persanes du «conte oriental» français. Et peut-être déjà quelques points à retenir : l'existence maîtrisée en Orient d'une parole miroir douée d'effets curatifs; la fragilité des moyens de transfert vers l'Occident, la vulnérabilité des traductions et des adaptations qui peuvent tantôt laisser échapper «la proie pour l'ombre», tantôt créer de nouvelles intrigues aux dépens d'un Orient soudain grotesque. 